\title{
On Motion, Its Relativity and the Equivalence Principle
}

\author{
Gabriel Barceló \\ Advanced Dynamics S.A., Madrid, Spain \\ Email: gabarce@iies.es
}

Received 31 August 2014; revised 26 September 2014; accepted 18 October 2014

Copyright (C) 2014 by author and Scientific Research Publishing Inc.

This work is licensed under the Creative Commons Attribution International License (CC BY).

http://creativecommons.org/licenses/by/4.0/

(c) (i) Open Access

\section{Abstract}

The Equivalence Principle put forward by Albert Einstein is currently undergoing comprehensive revision to determine its degree of accuracy. Notwithstanding, this principle refers to a very specific circumstance, as free-fall; thus in our opinion it cannot be generalised to any other movement in space. This paper refers to the dynamic hypotheses of moving rigid bodies and a particular, structured theory that would establish how such bodies behave when subject to different actions that oblige them to make successive, non-coaxial spins. With respect to bodies subject to acceleration by rotation, we understand that there are indications to identify the prior dynamic state of the moving object and that examples of a violation of the aforementioned Equivalence Principle can be deduced thereof. Based on the findings of this paper and the theory put forward herein, we suggest that an observer can identify the prior situation of absolute rest or absolute non-rotation of a body, thus leading to the conclusion that movement does not necessarily have to be a relative concept. The foregoing leads us to propose that the Equivalence Principle is fully valid for the situation put forward by Albert Einstein, but cannot be generalised to any dynamic situation.

\section{Keywords}

Equivalence Principle, Rotating Bodies, Non-Coaxial Spins, Dynamic Interactions

\section{The Equivalence Principle}

When Albert Einstein stated that, "We (...) assume the complete physical equivalence of a gravitational field and a corresponding acceleration of the reference system” [1], he was putting forward the Equivalence Principle, the grounds for his General Theory of Relativity and for the new concepts that he introduced into physics with respect to movement and space-time.

According to the original Equivalence Principle, as described by Einstein himself, the free-fall of a body in 
space subject to a gravitational field is physically equivalent to inertial movement. Many conclusions concerning body movement and mass can be deduced from this principle.

Indeed, numerous experiments and tests have been carried out to verify the Equivalence Principle, including testing the equivalence between gravitational mass and inertial mass.

There are several ongoing experiments using satellites to test the Equivalence Principle from space. For example: Satellite Test of the Equivalence Principle (STEP), GALILEO GALILEI and Micro-satellite pour l'Observation du Principe d'Équivalence (MICROSCOPE).

STEP is being carried out by Stanford University and an international team of collaborators. It will be capable of detecting any slight deviation from the Equivalence Principle at the level of 1 part in $10^{18}$.

The French programme, MICROSCOPE, will have two pairs of test masses and will be capable of detecting any violation of the Equivalence Principle with a sensitivity of 1 part in $10^{15}$. According to the initial programme, it is estimated that its findings will not be known or used until 2018.

The Italian experiment, GALILEO GALILEI, is of similar design, although it only uses one pair of test masses. To improve the accuracy of the experiment, the satellite will be turning on its central axis at a speed of 2 revolutions per second. The experiment should be capable of achieving a sensitivity of 1 part in $10^{17}$.

These experiments aim to test the universality of free-fall and to define the degree to which the Equivalence Principle and, where appropriate, General Relativity, are accurate.

Nevertheless, we believe that it is now possible to find new fields of research into the Equivalence Principle and possible violations of it. However, we do not know whether other authors have made similar contributions to those we are proposing in this paper.

\section{Research Project}

I have always been greatly interested in the behaviour of rotating bodies, and specifically in the case of a rigid body undergoing different, non-coaxial spins.

The dynamic systems in the universe are in constant motion, but also in constant dynamic equilibrium, free-fall being an exceptional and unusual phenomenon. We cannot but be struck by the dynamic harmony of the cosmos, which continues to maintain its apparent stability over time. At the very heart of this dynamic equilibrium there is a fact that stands out and is repeated and reiterated over time: celestial bodies are not only in orbit, they also have intrinsic rotation. It was speculations about this aporia or puzzle, together with the conjectures of Prof. Miguel A. Catalán [2] (see Figure 1), that led us to research this area.

Our initial speculations and conjectures:

A. Dynamic behaviour of rigid solid bodies in non-inertial systems:

- dynamic equilibrium: universe in constant dynamic balance;

- orbiting coexists with intrinsic rotation.

B. Prof. Miguel A. Catalán’s conjectures.

Over the last thirty years, we have conducted research with the aim of understanding the behaviour of rigid bodies that are experiencing simultaneous non-coaxial spins. This research has led us to propose new hypotheses

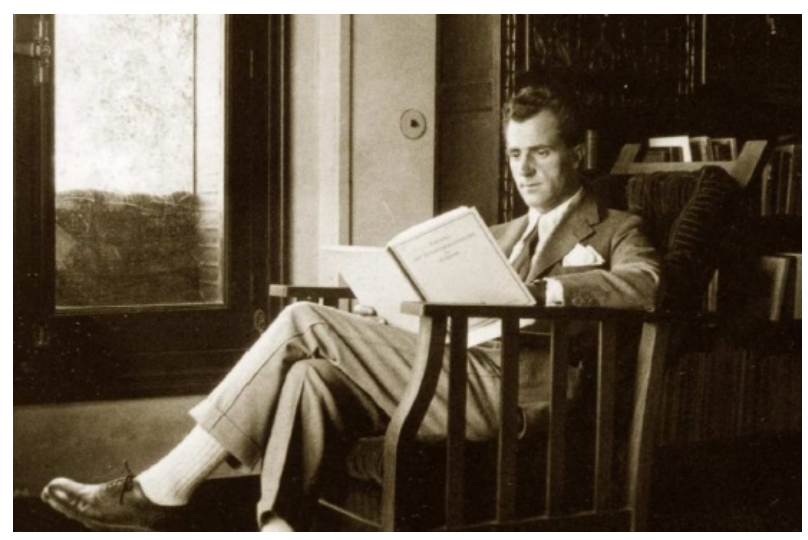

Figure 1. Miguel A. Catalán Sañudo, spectroscopist (Zaragoza; 1894 - Madrid; 1957). 
in the area of field theory to explain the dynamic behaviour of bodies that are subject to acceleration by noncoaxial spins.

Our initial aims in this project were:

- to analyze non-inertial systems;

- to research rotating bodies;

- to study the behaviour of rigid bodies experiencing simultaneous non-coaxial spins;

- to study inertial reactions: multiple rotations.

In studying the rotational dynamics of bodies, we have had to define exact terms for specific physical concepts. Accordingly, in this paper we refer to bodies endowed with Intrinsic Angular Momentum [3], in Constant Rotation, in which the Rotational Inertia generates Dynamic Interactions caused by a Coupling [4] or the non-discriminating algebraic addition of velocities. The concept of the Intrinsic Rotation of a body is equivalent to that of spin in quantum mechanics. All of these concepts have been developed in previous texts [5].

"We define the inertial reactions shown in matter when it is subjected to accelerations to be Dynamic Interactions (DI). These are shown in nature at all scales of magnitude. Any physical system and boundary conditions can be represented by a Lie group. The phase space of this dynamic is 8-dimensional and is described in a quaternionic Kähler variety of symplectic geometry. This would have two types of fields simultaneously: one corresponding to the actual forces applied, and the other corresponding to inertial forces due to the Dynamic Interactions (DI) generated" [6]. Our findings led us to propose the Theory of Dynamic Interactions (TDI), which can be consulted on several websites [7] and in several texts [8]. These sources explain how this theory has far-reaching effects on the basic principles of dynamics, not to mention cosmology [9], astrophysics [10], atomic physics and technology [11].

At the end of the 19th century, M. E. Jouffret had already begun to analyze the dynamic reactions caused in a particle of a rigid body when it is subjected to successive non-coaxial torques [12]. M. Hirn stated that a nonhomogeneous velocity distribution was generated in such situations [13].

In our studies, we have come to the conclusion that the dynamic behaviour of a rigid body moving in space is determined by the dynamic fields that are generated in it. In the case of simple rotation, the velocity field generated is isotropic. However, if the body is subjected to an additional rotation that is not coaxial with the first one, a new anisotropic velocity field is generated, as stated above. Figure 2 shows the velocity fields that are generated at the edge of a disc that is subject to rotation (homogeneous distribution) and those generated when it is subjected to two successive torques that are not coaxial (non-homogeneous distribution).

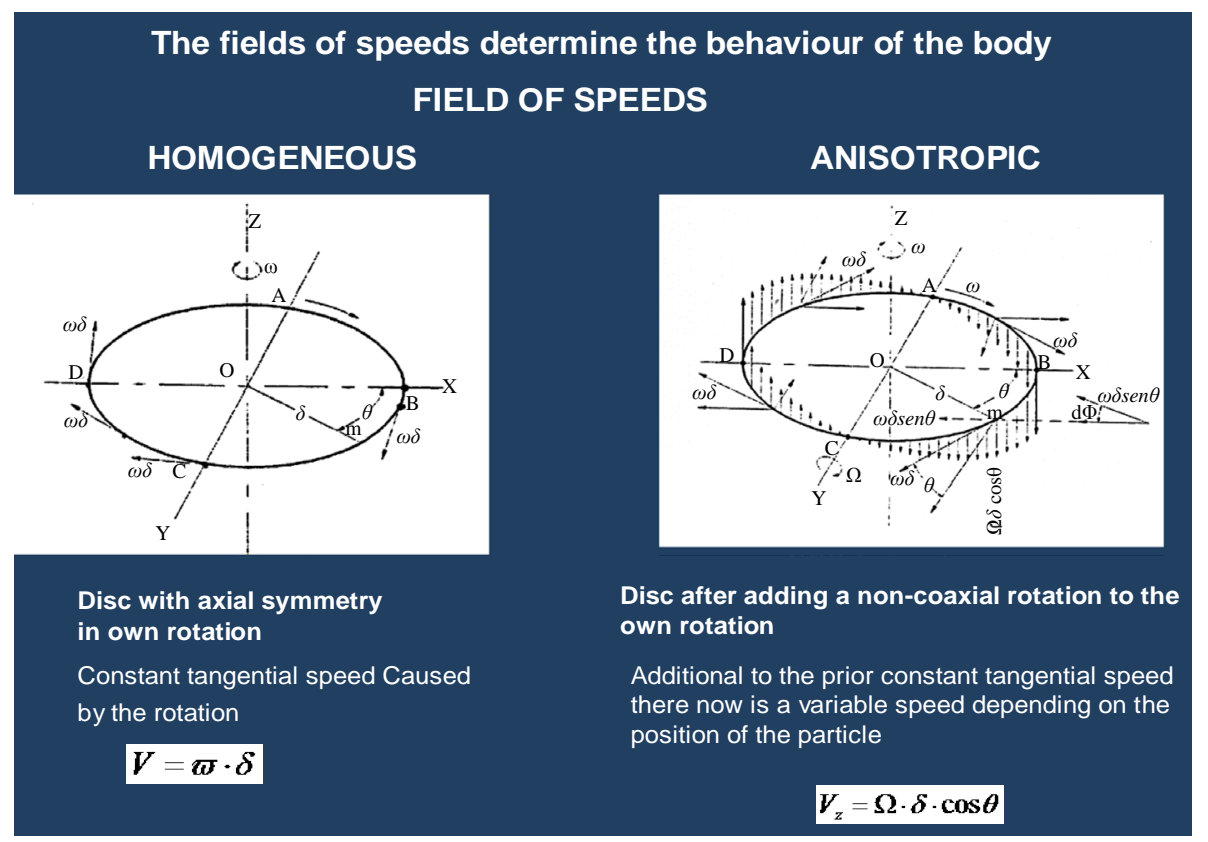

Figure 2. Comparative analysis of the velocity fields generated in rotation and by subjecting the body to two successive non-coaxial torques. 
These non-homogeneous fields generated in the moving object are what cause its change of path, since they cause it to change the direction of its translational speed. Figure 3 shows the velocity fields and accelerations of a body subjected to two successive torques, each applied to a different perpendicular axis.

Based on these studies, it is possible to both conceive of and structure a new rotational dynamics for non-inertial systems. This new rotational dynamics could answer important problems in physics that are as yet unresolved, or have hitherto been wrongly interpreted.

Based on these principles, we have created a rotational dynamics in which the translation velocity field is coupled, or vectorially added, to the anisotropic velocity field of the velocities generated by the second moment, remaining constant and independent of the inertial rotation velocity field, due to the Rotational Inertia. This way of understanding the dynamic behaviour of rotating bodies differs from the one that has been traditionally accepted in classical mechanics since Poinsot [14], which only allows for the coupling together of the movements caused by equivalent external moments.

In our proposal, we apply the criterion of superimposing movements through the non-discriminating coupling of velocity fields on each of the points on the moving body's mass, instead of the Poinsot criterion. According to our hypothesis, the translation velocity fields are algebraically added to the velocity fields generated by the second torques and, instead of a new rotation, the result is a movement in which the centre of mass of the moving body is orbiting while, at the same time, maintaining its initial intrinsic rotation. The path of the body's centre of mass therefore changes, without any external force being applied in that direction.

Certain axioms and laws of behaviour [15] for these hypotheses have been deduced from the principles of the conservation of total mass, total energy and the amount of movement.

An equation for non-differential movement has been deduced from this theory, according to which the translational velocity of the centre of mass of a body which is subjected to accelerations caused by successive, non-coaxial torques, is determined by a rotation in space of the initial translational velocity. This initial translational velocity of the moving body's centre of mass is defined by the matrix $\Psi$, as can be seen in Figure 4.

The proposed hypotheses and axioms not only fully explain the initial aporia or puzzle inherent in the fact that bodies in the observable universe both rotate and orbit, but also provide explanations of the behaviour of celestial bodies in general, not to mention the flight of the boomerang [16].

Videos have been created [17] that present this theory. Moreover, we have conducted several experiments which confirm it, and videos of these experiments can also be viewed [18]. Based on documents we have published in journals and conferences, other experiments have also been carried out by independent researchers [19] who are not connected to our team. These other experiments can also be viewed on the Internet [20].

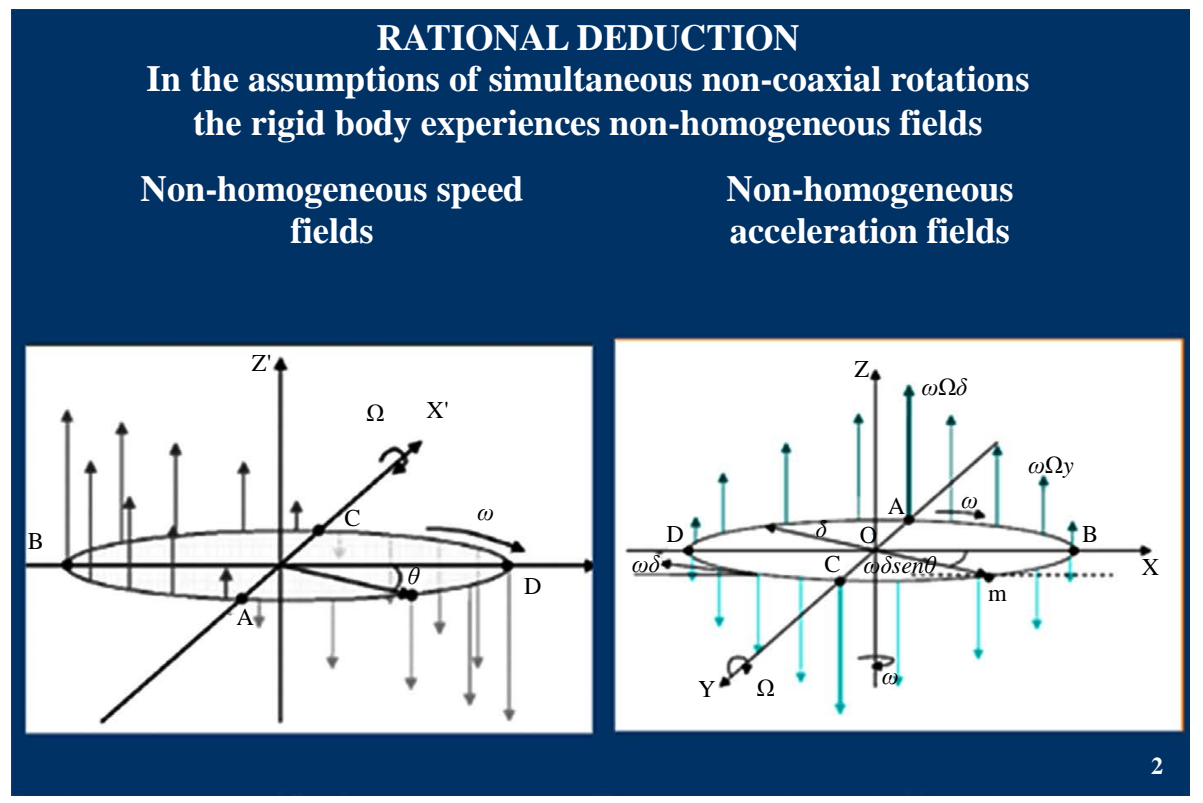

Figure 3. The velocity fields and accelerations that are generated in a body subjected to two successive non-coaxial torques are not homogeneous. 


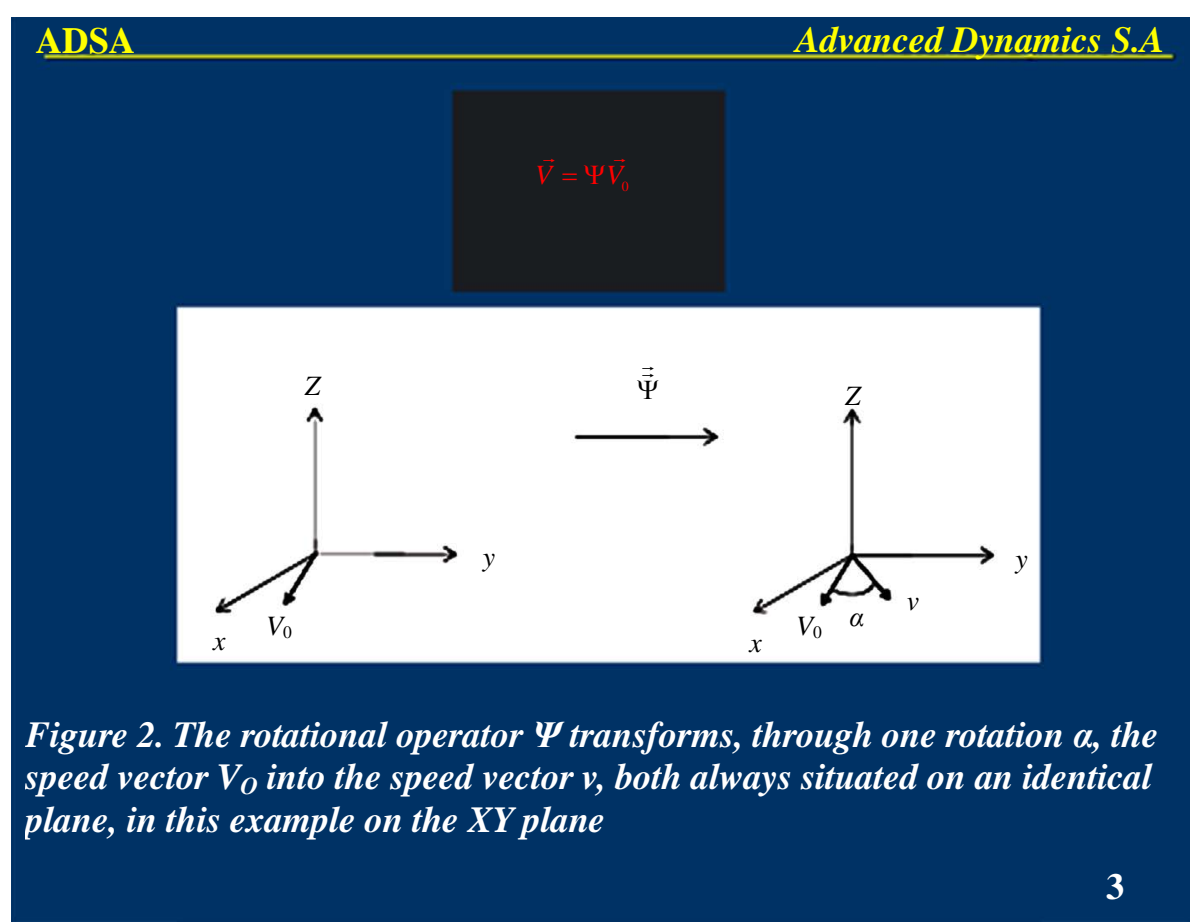

Figure 4. Movement equation.

\section{The Relativity of Motion}

Absolute space is a concept that was described by Newton [21] in order to give his laws of dynamics universal validity. This concept made it possible to conceive of a universal reference system that could be applied to any movement, and, at the time, it seemed to be logically indisputable.

However, nearly two hundred years later, the analysis and innovations contributed by Ernst Mach [22] to the field of mechanics and, specifically, his analysis of the nature of the inertia of bodies, led scientists to abandon the abstract concept of absolute space. Absolute space and time were, for Mach, concepts based on anthropomorphic assumptions that lacked sufficient scientific foundation, and were tantamount to considering the Earth to be the centre of the universe.

Since Einstein, movement has been understood to be a relativistic concept, a function of the observer's reference system. Different observers can use different reference systems. Therefore, in relating the observations made by different observers to each other, the movement of each reference system in relation to the other systems must be taken into account.

Clearly, if the concept of movement is relative, so is the concept of the absence of movement, i.e. the concept of rest. Based on these premises, conventional present-day physics believes that it is not possible to determine the absolute dynamic state of a body, given that this is always understood to be defined solely by its relative frame of reference.

\section{Absolute Rotation}

The rotation of a body that is rotating independently of any external reference, i.e. is in absolute rotation, has long been debated, and this will be analysed in this paper on the basis of the dynamic theory we have proposed. This analysis is conducted quite outside, and separately from, any possible controversy as to the existence of absolute time and space.

This absolute rotation is a question that interested Newton, who suggested several tests for detecting a body's possible intrinsic rotation. For example, in the case of a bucket filled with water, the geometry of the liquid surface, according to Newton, would enable us to determine whether it was the bucket or the observer that was rotating. If the bucket were rotating on its axis, the centrifugal force would have modified the supposedly flat surface of the water. The tension of a string joining two spheres that are rotating around their centre of mass can 
also be used to infer this rotation, once again on the basis of the effect of centrifugal force. An object's geometrical distribution was another argument often resorted to, using the Earth itself as a reference: its rotation could be detected if it were flat at the poles and bulged at its equator. This rotation could also be inferred from the shape caused by the secular equilibrium between gravitational attraction and the centrifugal force.

\section{Logical Deductions from the Theory of Dynamic Interactions}

Having given this brief historical analysis of the relativity of movement, we would like to suggest that, on the basis of the Theory of Dynamic Interactions (TDI) we are proposing, an observer could identify the dynamic state of a body in space, whatever the reference systems used. In fact, that is the conclusion we have reached. Moreover, it is possible to determine the following: the nature of the movement of a body in space; whether the system we are observing is subject to acceleration caused by a gravitational field or not; and whether or not we are observing a non-inertial, accelerated system.

We propose to conduct certain thought experiments to substantiate these claims. Let us suppose that we are observing a rigid body that has intrinsic rotation in space with respect to the observer. Even though the observer remains in a stationary position with respect to the moving body being observed, he will not, in principle, be able to know its absolute dynamic state.

However, we can subject that moving body to a torque that would cause it to spin on a different axis. In this case, in accordance with the hypotheses being proposed, certain dynamic reactions could occur, and the observer may perceive one of two new situations:

- the rigid body acquires a new intrinsic rotation that coexists with the existing non-coaxial one, but does not change its relative position in space; or

- the path of the body changes in relation to the observer, and it does not acquire any new intrinsic rotation.

In the first case, the observer can infer that the observed body does not move in space-i.e. it has zero intrinsic translational speed.

In the second case, the observer may conclude that the observed body has translational speed, and therefore, that both are moving in space. In addition, if the moment remains constant and the body launches into a closed orbital path, the observer can even infer that the translational speed is constant (Figure 5). Moreover, if the body enters a spiral path, the observer can infer that its own translational movement is accelerated (Figure 6). Indeed,

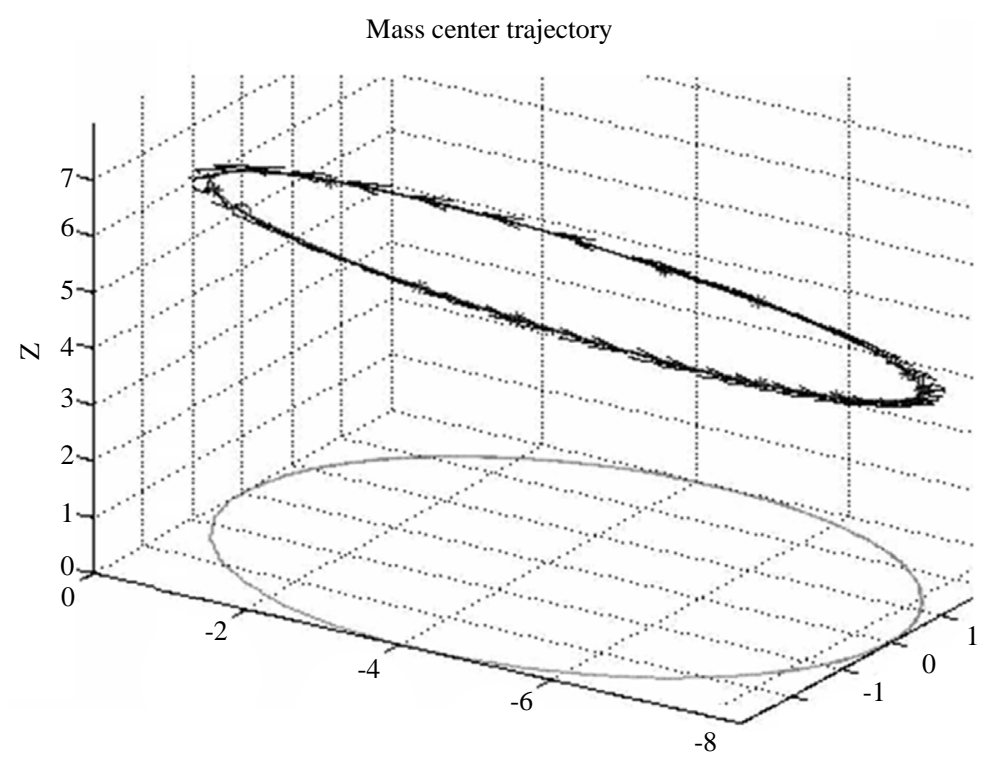

Figure 5. TDI path of the centre of mass of a moving object with intrinsic rotation that is simultaneously subjected to an external moment that is not coaxial with its intrinsic angular momentum. This path was obtained via computer simulation for the hypothetical case that both the applied moment and the translational linear speed of the moving object are constant. Simulation conditions: tangential speed $5 \mathrm{~m} / \mathrm{s}$. 


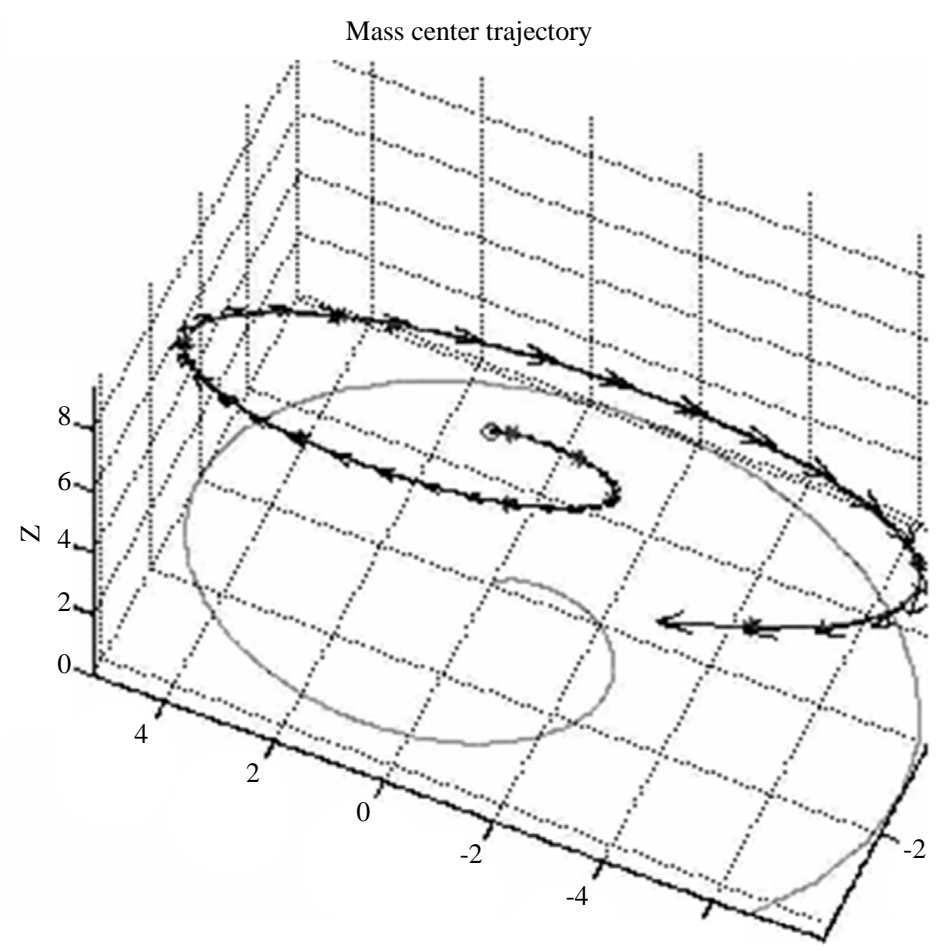

\begin{abstract}
Figure 6. Path of the centre of mass of a moving object, in the hypothetical case that the applied moment is constant, but the translational linear speed of the moving object is changing. This was obtained via computer simulation, based on the mathematical model of dynamic interactions. Tangential speed changes according to $\mathrm{S}=5+0.2 * \mathrm{t}(\mathrm{m} / \mathrm{s})$. Constant torque, always perpendicular to the tangential speed vector.
\end{abstract}

it is even true that he is able to distinguish whether the body was decelerating or accelerating, depending on whether the trajectory of the body observed is in an inward or outward spiral [23].

This logical example shows us that it is possible to identify the prior dynamic state of a body in space, and state whether it was moving or immobile, regardless of the systems of reference.

We can also analyse the rotational state of a moving object that is under observation, regardless of whether or not we can perceive its possible intrinsic rotation. We can subject the said body to successive non-coaxial torques, and if it changes its path when the first torque is applied, we can logically deduce that the body had prior intrinsic rotation, as well as translational speed. If the change of path occurs after the second, non-coaxial torque is applied, the body initially had no intrinsic rotation, but it did have translational speed. It was in a state of absolute zero rotation. Lastly, if, in this second case, the body does not change its path with respect to the observer, this means that initially it did not have either intrinsic rotation or initial speed: the body was at absolute rest.

It can be seen how, by means of these thought experiments, it is possible to identify the dynamic state of a body being observed, regardless of its systems of reference.

This procedure of logical deduction will also enable us to determine whether the body is subject to acceleration as a result of a gravitational field, or is a non-inertial, accelerated system. In the former case, the body's acceleration will be constant as it moves towards the attracting mass. In the latter case, applying successive non-coaxial torques will cause the moving body to follow a spiral course.

Clearly, for our proposal about the concept of absolute rotation to be scientifically significant, the necessary experiments should be carried out to verify our logical deductions from the thought experiments. According to our reasoning, we could identify whether an object is stationary or rotating on its own axis, or if it is the fact that the observer is moving that leads us to this possible deduction, thus overcoming the conceptual barrier represented by the relativity of motion.

We also suggest that the dynamic tests and experiments to check the validity of the Equivalence Principle should be conducted in a general, natural setting and not in the specific case of free-fall. Indeed, it is possible 
that in those other settings we may find a significant exception to the Equivalence Principle.

\section{Conclusions}

We have analysed the physical phenomenon of a rigid body that is subject to multiple rotations around different axes, and deduced the laws governing its behaviour and their mathematical expression, which is a rotation matrix.

Classical mechanics made it possible to create a mathematical model of an inertial, physical world, and provided satisfactory results. However, with respect to rotational dynamics, it fails to give us a suitable structured conceptual context, since it demands the use of analogies or references to specific dynamic effects, such as that of the gyroscope.

Relativity represented a major advance in our understanding of the dynamics of accelerated bodies. It may, however, be extended for certain cases, such as that of a body subjected to successive actions that result in noncoaxial spins.

In such cases, we suggest a clearly structured rotational dynamics, based on conservation principles and welldefined axioms, such as those put forward in the Theory of Dynamic Interactions.

The logical deductions proposed are based on the hypotheses of the theory being put forward, which in turn is founded on the coupling of the fields of the translational velocities at each point on the moving object, whether these fields are caused by translational movements, or are generated by the action of forces that are non-coaxial with the moving body's pre-existing intrinsic rotation.

In our opinion, applying field theory makes it possible to discover new areas of research into rotational dynamics. These new criteria involving the coupling of fields of velocities at points in rotationally accelerated systems have been confirmed by experiments and also simulated by means of a mathematical model.

We believe that these results suggest new horizons for rotational dynamics and new keys to understanding the harmony of the universe. This is a universe made up not only of forces, but also of their moments, which, when they act constantly on rotating rigid bodies that also have constant translational speed result in movement in a closed orbit. This is therefore a system in movement, albeit one in a constant state of equilibrium.

Indeed, is this not the equilibrium of the cosmos and the behaviour of the celestial mechanics that we observe?

We consequently believe that the mathematical model we are proposing is of clear importance. Moreover, we believe that a model is necessary in order to understand the dynamics of rotating bodies, and specifically, if we are to understand the dynamics of the cosmos, with bodies in orbit and with constantly recurring movements that make it possible for systems that are in centuries-long dynamic equilibrium and not in steady, limitless expansion. We further believe that this new theory of dynamics will afford us a greater understanding of the universe and the matter of which it is made. We have seen how, in the cases of bodies subjected to accelerations by rotation, it is possible to find ways of identifying the moving object's previous state, and that from these, violations of the Equivalence Principle can be deduced.

An observer can identify the dynamic state of a body in space and even whether or not it was previously in a state of absolute rest or absolute absence of rotation, and this leads us to the conclusion that movement does not necessarily have to be a relative concept. We have come to this conclusion by means of logical abstraction, whereby an observer can identify the dynamic state of the moving object under observation by its responses, but we believe that this logical deduction can be experimentally validated, and will thus confirm the true nature of the movement of matter.

We also propose that the Equivalence Principle is fully valid for the situation put forward by Albert Einstein, but cannot be generalised to all other cases of moving bodies.

We suggest a detailed and in-depth study made of the hypotheses we are putting forward, and we seek to have the requisite experiments conducted to confirm them. This would require experiments to be performed on models in orbit having intrinsic rotation.

\section{References}

[1] Einstein, A. (1907) Über das Relativitätsprinzip und die aus demselben gezogene Folgerungen, Jahrbuch der Radioaktivitaet und Elektronik. [On the Relativity Principle and the Conclusions Drawn from It. In: The Collected Papers of Albert Einstein. Vol. 2: The Swiss Years: Writings, 1900-1909, Princeton University Press, Princeton.]

[2] Barceló, G. and Catalán Sañudo, M.A., Eds. (2012) Arpegio. Sant Cugat. Chap. XIV. 
[3] Landau, L.D. and Lifshitz, E.M. (1976) Mechanics. 3rd Edition, Volume 1 (Course of Theoretical Physics), Butterworth-Heinemann, Oxford.

Landau, L.D. and Lifshitz, E.M. (1994) Mecánica. Editorial Reverté S.A., Argentina, 24.

[4] Goldstein, H., Poole, C. and Safko, J. (2000) Classical Mechanics. 3rd Edition, Addison-Wesley, Boston.

[5] Barceló, G. (2005) El vuelo del bumerán (The Flight of the Boomerang). Editorial Marcombo, Barcelona.

[6] Barceló, G. (2010) On the Equivalence Principle AC-10-A.2.1.1. 61st International Astronautical Congress, Prague. http://www.coiim.es/forocientifico/FORO\%20CIENTFICO/Documentos/ON_THE_EQUIVALENCE_PRINCIPLE.pdf Barceló, G. (2011) Analysis of Dynamic Field Systems Accelerated by Rotation. Dynamics of Non-Inertial Systems. DeMSET-2011 Congress, Miami.

http://www.coiim.es/forocientifico/FORO\%20CIENTFICO/Documentos/DeMSET_2011_GBarcelo.pdf

[7] Advanced Dynamics S.A.

http://advanceddynamics.net/en/

http://www.youtube.com/watch?v=vSUkd4slHGQ

http://www.youtube.com/watch?v=P9hGgoL5ZGk\&feature=c4-overview-vl\&list=PL3E50CF6AEBEED47B

http://www.dinamicafundacion.com/

[8] Barceló, G. (2012) World Journal of Mechanics, 2, 175-180.

http://dx.doi.org/10.4236/wjm.2012.23021

[9] Barceló, G. (2013) Imago Universi: A Story of the Human Conception of the Cosmos. Ed. Arpegio, Barcelona. http://www.editorialarpegio.com/ http://imagouniversi.com/

[10] Barceló, G. (2013) International Journal of Astronomy and Astrophysics, 3, 385-391. http://dx.doi.org/10.4236/ijaa.2013.34044

[11] Barceló, G. (2013) Global Journal of Researches in Engineering, 13. https://globaljournals.org/GJRE_Volume13/E-Journal_GJRE_(G)_Vol_13_Issue_5.pdf

[12] Jouffret, M.E. (1874) Théorie élémentaire des phénomènes que présentent le gyroscope, la toupie et le projectile oblong. Revue d’Artillerie, Berger-Levrault et Gauthier-Villars, Paris.

Gilbert, P. (1876) Problème de la rotation d'un corps solide autour d'un point. Annales de la Société Scientifique de Bruxelles, 316.

[13] Hirn (1876) Théorie analytique élémentaire du gyroscope. In: Gilbert, P., Ed., Problème de la rotation d'un corps solide autour d'un point, Annals de la société scientifique de Bruxelles, Bruxelles.

[14] Poinsot, L. (1878) Théorie nouvelle de la rotation des corps, 1834. In: Gilbert, P., Ed., Problème de la rotation d'un corps solide autour d'un point fixe, Annals de la société scientifique de Bruxelles, Bruxelles, 258.

Poinsot, L. (2005) Théorie nouvelle de la rotation des corps, 1834. In: Barceló, G., Ed., El vuelo del bumerán (The Flight of the Boomerang), Marcombo, 121.

[15] Barceló, G. (2013) World Journal of Mechanics, 3, 328-338. http://dx.doi.org/10.4236/wjm.2013.39036

[16] Barceló, G. (2014) Journal of Applied Mathematics and Physics, 2, 569-580. http://www.scirp.org/journal/jamp http://dx.doi.org/10.4236/jamp.2014.27063

[17] Bauluz, E. New Dynamic Hypotheses. www.advanceddynamics.net http://vimeo.com/62601974 http://dl.dropbox.com/u/48524938/VTS http://www.youtube.com/watch?v=vSUkd4slHGQ\&feature=c4-overview\&list=UUgDHgaGi2I2rmZNoanNbVWQ

[18] Theory of Dynamic Interactions. http://www.youtube.com/watch?v=P9hGgoL5ZGk\&list=PL3E50CF6AEBEED47B http://www.youtube.com/watch?v=XzTrGEtJGXU\&list=PL3E50CF6AEBEED47B http://www.youtube.com/watch?v=dtMqGSU9gV4\&list=PL3E50CF6AEBEED47B http://www.youtube.com/watch?v=qK5mW2j2nzU\&list=PL3E50CF6AEBEED47B

[19] Pérez, L. (2013) World Journal of Mechanics, 3, 174-177. http://www.scirp.org/journal/wjm http://dx.doi.org/10.4236/wjm.2013.33016

[20] Pérez, L.A. (2013) Reflecting New Evidence on Rotational Dynamics. http://vimeo.com/68763196

[21] Newton. Principia, Im. Du Chasteller, Paris, Proposition 2, 1757.

[22] Mach, E. (1921) Die Mechanik in Ihrer Entwicklung Historisch-Kritisch Dargestellt. Leipzig, Brockhaus.

[23] Barceló, G. (2010) On the Equivalence Principle AC-10-A.2.1.1. Proceedings of the 61st International Astronautical Congress, Prague, 27 September-1 October 2010. 
Scientific Research Publishing (SCIRP) is one of the largest Open Access journal publishers. It is currently publishing more than 200 open access, online, peer-reviewed journals covering a wide range of academic disciplines. SCIRP serves the worldwide academic communities and contributes to the progress and application of science with its publication.

Other selected journals from SCIRP are listed as below. Submit your manuscript to us via either submit@scirp.org or Online Submission Portal.
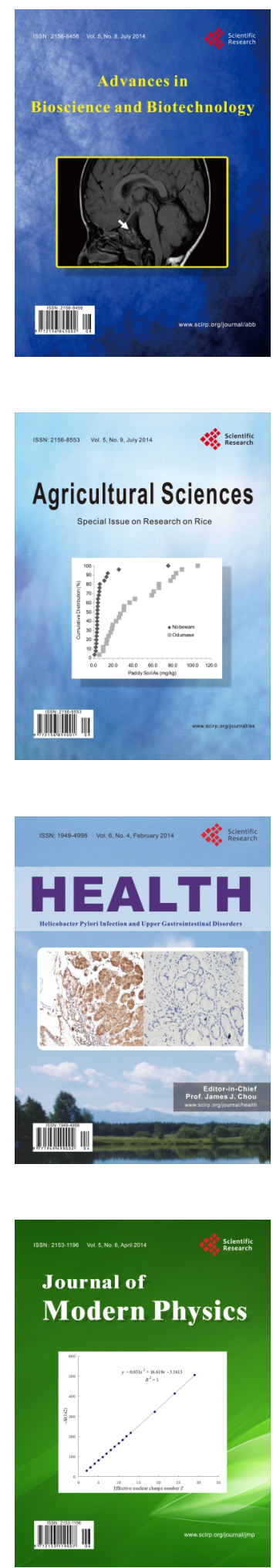
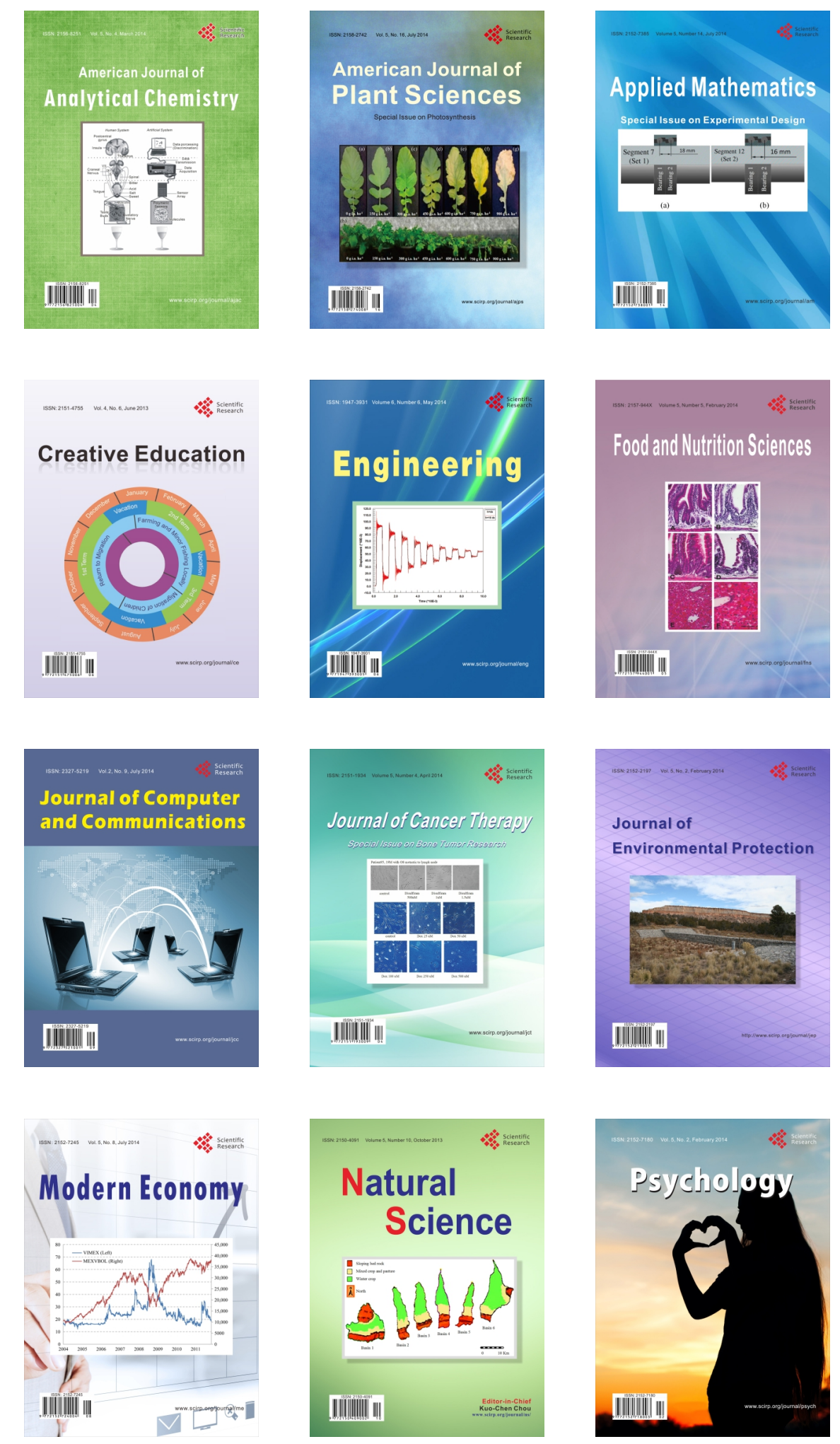